\title{
Teoría fundamentada y sus implicaciones en investigación educativa: el caso de Atlas.ti
}

\author{
José Eduardo Padilla Beltrán ${ }^{1}$ \\ Paula Lizette Vega Rojas ${ }^{2}$ \\ Diego Armando Rincón Caballero ${ }^{3}$
}

\begin{abstract}
Resumen
Durante los procesos de investigación formativa, los grupos, líneas, semilleros e investigadores que realizan estudios conforme a un enfoque cualitativo dirigido al análisis de información, se ven abocados a utilizar estrategias y software para el análisis de datos cualitativos, sin embargo, el uso indiscriminado del apoyo tecnológico en ciertos momentos subordina la capacidad del investigador para discernir o destacar el componente teórico. Por ello, se pretende dar cuenta, por medio de un enfoque descriptivo, de la importancia de un sustrato teórico que oriente el apoyo tecnológico, en tanto se ejemplifiquen métodos de comparación constante, abierta o axial, de gran relevancia en la teoría fundamentada, cuya implicación devela la acción del investigador por conocer los referentes metodológicos y epistemológicos que respaldan la acción del software en la investigación a desarrollar. En ese orden de ideas, resaltar la acción teórica y, en el caso de la teoría fundamentada, la parte interpretativa, documental y explicativa llevada a cabo por los investigadores para consolidar los datos de forma rigurosa, es sinónimo de una investigación que aprovecha los recursos tecnológicos para dar paso a una labor acuciosa en la caracterización y relación de categorías de análisis y emergentes.
\end{abstract}

Palabras clave: análisis de datos, Atlas.ti, formación docente, tecnología CAQDAS, teoría fundamentada.pedagógicas.

\footnotetext{
1. Licenciado en Matemáticas, Universidad Pedagógica Nacional; Especialista en Educación, Universidad Manuela Beltrán; Magíster en Administración y Supervisión Educativa, Universidad Externado de Colombia; Magíster en Educación con énfasis en Evaluación Educativa, Universidad Santo Tomás; Doctor en Educación, Newport University, USA. Director del Centro de Investigaciones de la Facultad de Estudios a Distancia de la Universidad Militar Nueva Granada; Líder del Grupo de Investigación Pedagogía y Didáctica en la Educación Superior (PYDES).

Correo: eduardo.padilla@unimilitar.edu.co

2. Relaciones Internacionales y Estudios políticos, Universidad Militar Nueva Granada. Joven Investigadora del Grupo PYDES.

Correo: vega527@gmail.com
}

3. Licenciado en Diseño Tecnológico, Universidad Pedagógica Nacional; Estudiante de Maestría en Educación, Universidad Pedagógica Nacional. Asistente de Investigación del Grupo PYDES.

Correo: diego.rincon@unimilitar.edu.co 


\title{
Grounded theory and its implications for educational research: the case of Atlas.ti
}

\begin{abstract}
During formative research processes, groups, lines, seedlings and researchers who study according to a qualitative approach aimed at analyzing information, are forced to use strategies and software for qualitative data analysis, however, the indiscriminate use technological support at times makes the researcher's ability to discern and highlight the theoretical component. Therefore, it seeks to explain through a descriptive approach to the importance of a theoretical foundation to guide technology support, as they exemplify constant comparison methods, open or axial, of great importance in grounded theory, the importance of which reveals the action of the researcher to know the methodological and epistemological references supporting software in action research to develop. In that vein, highlight the theoretical action and, in the case of grounded theory, the interpretive, explanatory documents and conducted by researchers to consolidate data in a rigorous, is synonymous with research that draws on the resources technology to make way for a diligent work on the characterization and relationship analysis and emerging categories.
\end{abstract}

Keywords: data analysis, Atlas ti, teacher training, technology CAQDAS, grounded theory.

Recibido: 09-03-2014

Aceptado: 25-04-2014

\section{Introducción}

El análisis de datos cualitativos en el campo de las investigaciones educativas, ha tenido mucho auge en lo que va del siglo XXI, pues se compenetra con soportes tecnológicos de tipo Computer Assisted Qualitative Data Analysis Software [CAQDAS] (Software para el análisis de datos cualitativos asistidos por ordenador), más específicamente el software Atlas.ti. (Chacón, 2004), en aras de realizar análisis complejos de una manera sistemática y ordenada al facilitar la labor del investigador. Sin embargo, el problema radica en la carencia -en algunos estudios- de un referente claro de análisis entre las relaciones de teoría, metodología y objetivos que lleven a un nivel de abstracción capaz de desarrollar 
unas proposiciones teóricas en el ámbito de la investigación social (Sautu et al., 2005). De igual forma, se presenta el uso indiscriminado y dependiente de la herramienta o software, sin tener conocimiento sobre los preceptos teóricoconceptuales que le relacionan con las habilidades del investigador y su conocimiento experto sobre el tema a investigar.

En esa medida, el presente escrito trata de dar cuenta de una tendencia, sobre el nivel de coherencia, que puede existir cuando se estudia a profundidad la teoría que respalda el análisis de datos cualitativos, en este caso particular; la teoría fundamentada expuesta por Strauss y Corbin (2002), y las raíces históricas en el marco de la sociología aplicada a la investigación educativa desde el interaccionismo simbólico y el uso de Atlas.ti, como una herramienta de innovación en el análisis cualitativo (Valles, 2002).

A la luz de caracterizar esta vinculación teórico-práctica de la teoría fundamentada y el uso del software Atlas.ti, se pretende como objetivo principal establecer didácticamente un proceso de conceptualización y puesta en práctica de la teoría fundamentada -sin pretensión de construcción teórica- con apoyo mediático del software Atlas.ti., a través de una investigación realizada por el grupo de investigación: Pedagogía y Didáctica en la Educación Superior [PYDES] acerca de la formación pedagógica en el uso de las Tecnologías de la Información y la Comunicación [TIC] en el marco de la educación superior, involucrando la importancia y el estatuto de la teoría en la investigación social y el análisis de revisión documental para caracterizar elementos de emergencia en fuentes teóricas y acervos conceptuales sustanciales para el desarrollo de categorías emergentes. Así pues, la metodología del trabajo implica una subordinación teórica como punto de partida en la descripción de los procesos de clasificación, codificación y análisis de la información esenciales en el análisis de datos cualitativos soportados en tecnologías CAQDAS.

\section{Relaciones teóricas y metodológicas en los estudios de investigación social}

Dentro de los supuestos epistemológicos, ontológicos y axiológicos particulares de la investigación social, se halla imperante el entender las maneras de aproximarse a un conocimiento, que si bien en épocas contemporáneas bajo esa dimensión social se vuelve más complejo, se hace además inconmensurable determinar la importancia de una visualización teórica orientada a los preceptos empíricos, que la validan y la confrontan, en el marco científico a críticas y desarrollos de articulación con otras teorías (Sautu et al., 2005). En ese orden de ideas, en una investigación social y más aún en el campo educativo, se debe desarrollar un análisis profundo sobre el paradigma y el fundamento teórico y metodológico implicado para observar, analizar y describir la realidad. 
Los supuestos y bases de posicionamiento paradigmático brindan una perspectiva que ayuda a dimensionar, delimitar y contextualizar el problema como construcción del investigador, de igual manera, contribuye a que los objetivos sean claros a raíz de la cohesión entre lo teórico del fenómeno social y las acciones metódicas para abordar su justificación y comprobación. Por ello, en este caso, la investigación mediada por una teoría fundamentada, no solo confiere elementos metodológicos para conocer algunas posturas de otras voces sobre el objeto de estudio, también aproxima al investigador a cuestionarse y a desentrañar inquietudes sobre lo que se ha de investigar, esto repercute en lo que Sautu et al. (2005) denominan como «teoría general» y su especificidad en la «teoría sustantiva» (p.34), como mediadores entre objetivos específicos y lo acorde a las decisiones que se han de considerar para diseñar la selección de métodos y técnicas de recolección y análisis de datos.

Los objetivos en esta mirada teórica, son el puente de conexión para determinar una metodología concurrente a procesos metódicos enfáticos en dar cuenta de la existencia, permutación, exploración o descripción empírica del objeto de estudio. En ese sentido, es importante resaltar lo afirmado por Sautu et al. (2005) acerca de la diferenciación de la metodología y el método; mientras la primera es una cuestión eminente de -logos-, la segunda se encuentra adscrita a un camino u orientación que el investigador recorre para determinar el impacto de la investigación realizada. Para el caso de la investigación social y, en especial mención la desarrollada en el campo educativo, la metodología en gran parte se da en lo cualitativo, presentando métodos variados asociados a lo etnográfico, el discurso, el referente cultural y los grupos focalizados (Sautu et al., 2005: 47) como procesos que llevan al investigador a diseñar y articular técnicas que deben responder a dicho enfoque de investigación.

\section{Aproximación al referente de teoría fundamentada}

Como parte de la investigación de corte cualitativo, la teoría fundamentada ofrece una forma de representación del mundo. Este tipo de teoría se caracteriza por originarse a partir de la recolección y análisis sistemáticos de datos. Aquí la teoría tiene un papel importante, que es el de mostrar una serie de unidades de análisis concomitantes con datos observados por el investigador sobre algún tipo de fenómeno, mediante el análisis práctico de datos que surgen de la teoría. Esto permite que las tendencias emergentes de los datos coincidan más con la realidad y así ampliar el conocimiento hacia un tipo de acción significativa (Strauss y Corbin, 2002).

Este factor de emergencia hace que se empleen unas estrategias características, aunque no exclusivas de ella; primero, la recolección de datos y el análisis transcurren de manera concurrente al nivel de significancia otorgado por el investigador -teoría general: interaccionismo simbólico-; segundo, los datos 
determinan los procesos y productos de la investigación y no marcos teóricos preconcebidos -teoría sustantiva: emergente-; tercero, los procesos analíticos suscitan el descubrimiento y desarrollo teórico-conceptual y no la verificación de teorías ya conocidas -concepciones y orientaciones de la investigación-; cuarto, el muestreo se realiza con base en lo que emerge de los datos, se le denomina muestreo teórico que sirve para refinar, elaborar y completar las categorías; y por último, el uso sistemático de los procedimientos analíticos lleva a niveles más abstractos de análisis (Charmaz, 1990 citado en Strauss y Corbin, 2002).

Así, la teoría fundamentada crea conceptos y descriptores valiéndose directamente de los datos, propensos de teorías generales y sustantivas (Sautu et al., 2005). De manera inductiva, la teoría se va consolidando a partir de un estudio emergente, en el cual no hace uso de categorías generales aplicadas al objeto de estudio, sino que es desde la realidad y un problema que se van seleccionando y significando las categorías. Asimismo, según Strauss y Corbin (2002), la teoría fundamentada se apoya en aspectos metódicos en dos aspectos generales que son; el método comparativo constante que codifica y analiza los datos de manera simultánea, al posibilitar la creación de conceptos «Realizando una comparación continua de incidente con incidente, identifica sus propiedades, explora sus interrelaciones y logra integrarlo en una teoría que guarda una lógica razonable» (Giraldo, 2011: 2), y el muestreo teórico que evidencia nuevos elementos para contribuir a la ampliación de los conceptos y la tendencia de análisis del investigador.

Este juega un papel importante, en tanto él es quien tiene a la mano la experiencia y nivel de inferencia sobre el objeto de estudio - para la teoría fundamentada, se necesita que el investigador tenga un referente idóneo sobre el objeto de estudio; es decir, lo haya investigado bajo otra problemática o situación contextual-, y es desde sus concepciones y percepciones de la realidad, que las categorías emergentes de los datos analizados van tomando su significado frente a los objetivos de la investigación social. De este modo, una comparación constante entre categorías va permitiendo evidenciar la relación que hay entre ellas y es desde esa relación emergente, la tendencia teórica que explica el fenómeno estudiado (Hernández et al., 2011). 


\section{Estructura de la teoría fundamentada}

\section{Muestreo teórico}

Este muestreo se caracteriza por hacer de forma simultánea la recolección de datos y su análisis en relación a una teoría general y sustantiva (Sautu et al., 2005), permitiendo que los conceptos se vayan ampliando a lo largo de la investigación. Adicionalmente, en palabras de Javier Murillo:

Es importante tener en cuenta que el diseño de investigación es en espiral, es decir que a medida que la investigación avanza se conoce qué tipo de grupos, informantes o nuevos escenarios que deben explorarse para lograr la progresiva densificación y emergencia de teoría formal (s.f.: 10).

Este tipo de muestreo permite que desde las categorías iniciales surjan subcategorías consecuentes a profundizar, en la dimensión y relación del fenómeno estudiado, con otros aspectos o perspectivas teóricas. Así pues: «El muestreo se utiliza como procedimiento de comprobación del marco teórico, y no como verificación de hipótesis» (Murillo, s.f.: 12). A medida que se va desarrollando este muestreo, el investigador va identificando unas categorías globales que deben ser alimentadas para comprenderlas, de ahí la necesidad de ir creando otras sub-categorías para su descripción y nivel de afectación sobre el estudio. Así pues, la rigurosidad de este tipo de muestreo depende del proceso de análisis que haga el investigador, con el fin de dar respuesta al problema de investigación y no perder el horizonte metodológico ante la cantidad de conceptos que se van abriendo durante la recopilación de datos.

\section{Método comparativo constante}

Como su nombre lo dice, este aspecto se basa en la comparación constante de información, con el objetivo de ir dando forma a la tendencia teórica e ir depurando conceptos y significados a partir del rastreo de categorías y sus subcategorías. Igualmente, de manera simultánea se continúa con la codificación de los datos y su análisis. En suma, Hernández et al. (2011) afirman que:

Se trata entonces de ir paralelamente combinando en el análisis de los datos cualitativos, conceptos, categorías, propiedades e hipótesis interrelacionadas, teniendo presente la posibilidad del surgimiento de nuevas categorías con sus propiedades, las cuales deben ser incorporadas en el proceso de análisis (p. 10).

\section{Codificación abierta}

Se inicia cuando el investigador procede a dividir los datos, con el fin de hacer visible las categorías emergentes que se dan de los conceptos generales e iniciales de la investigación, es decir del muestreo teórico estudiado anteriormente. 
Asimismo, estas categorías emergentes hacen posible comprender el fenómeno, ya sea en sus causas, en su función, en sus consecuencias, en su proceso, entre otras. Este proceso se lleva a cabo por los investigadores para ir esclareciendo de manera teórica cada una de las partes que constituyen las categorías generales. Con la consolidación de información, el investigador mediante su análisis va abstrayendo nuevos conceptos, preguntas, hipótesis, implicaciones teóricas que amplían el conocimiento y comprensión de los datos. Por ende y en palabras de Strauss y Corbin citados en Murillo (s.f.):

De forma general, la codificación abierta: descompone los datos en parte discretas, se examinan minuciosamente y se comparan en busca de similitudes y diferencias. Los acontecimientos, sucesos, objetos y acciones o interacciones que se consideren conceptualmente similares en su naturaleza o relacionados en el significado se agrupan bajo conceptos más abstractos, denominados categorías (p. 24)

\section{Metodología}

De forma didáctica el presente apartado intenta desarrollar un abordaje metodológico de la teoría fundamentada, con las condiciones particulares del caso, a través de un estudio de investigación formativa parcial, acerca de la Formación docente en el uso de las TIC en procesos virtuales de aprendizaje 4 y cómo a partir de su relación teórica y metodológica se va incentivando el uso de ayudas tecnológicas tipo CADAS (Atlas. Ti), se puede generar un apoyo sustancial, siempre y cuando, como se ha venido recalcando, sea resultado de una acción consciente y pertinente a los referentes teóricos y metodológicos de la investigación.

Teniendo en cuenta la complejidad del análisis de los datos cualitativos, La investigación adopta un enfoque cualitativo (Sautu et al., 2005; Valles, 2003), para establecer relaciones o tendencias socio-demográficas y datos duros provenientes del análisis de la revisión documental. En ese sentido, como refieren los autores anteriormente citados, este abordaje permite: obtener datos más consistentes y por consiguiente una mayor multiplicidad de perspectivas sobre el estudio y sus analizadores. En este tipo de metodología, se consideran varias fuentes o documentos primarios acordes al objeto de estudio. A nivel de tratamiento de datos emergentes de la teoría general y sustantiva (Sautu et al., 2005) se recurrirá a la comparación constante (Valles, 2002), lo que confiere mayor fiabilidad y pertinencia de los resultados, posibilitando una fundamentación del análisis de datos efectuado con el software Atlas.ti.

Desde la metodología cualitativa se establece un esquema emergente (Glaser \& Strauss, 1967), empezando por la codificación abierta de los documentos -revisiones para el marco teórico-, sensible de ser clasificada, interpretada y 
organizada para un desarrollo teórico proveniente de múltiples datos (delimitados por el problema de investigación). De igual manera, la eventualidad emergente permitió construir en este estudio, una relación dialógica entre la literatura de formación docente con uso de TIC y las implicaciones de las TIC para formar docentes en educación superior.

Desde el proceder cualitativo, también se lleva a cabo un análisis descriptivo de las literaturas consultadas, es decir, la proveniencia o el país del estudio y el número de documentos por su tipología (artículos, tesis de posgrado, libros producto de investigación y conferencias). En ese orden de ideas, la parte de fiabilidad de los textos primarios se apoya en una relación demográfica y a la vez desde: la fundamentación de la categoría (en el software es igual a conocer el número de citas por código), y la densidad de relación de sub-categorías (en Atlas.ti se traduce en la cantidad de relaciones conceptuales entre códigos) para desarrollar un esquema de concurrencias con apoyo del software.

\section{Primera etapa}

Se desarrolla una base de datos con textos acordes a la conceptualización y formación de docentes en lo concerniente a las TIC, en tanto esta información sirve como banco principal para el acervo de documentos primarios a utilizar en el análisis y clasificación de los datos cualitativos en el software Atlas.ti. Igualmente, los criterios para la selección de los textos fueron: la pertinencia con el tema, la relación con la hipótesis, actualidad del estudio y el rigor conceptual denotado en el texto.

\section{Segunda etapa}

Retomando la etapa uno del proceso metódico, se prosigue a una depuración y clasificación de los documentos, por lo cual se hizo necesario establecer diálogos entre los integrantes del grupo para acordar un consenso de los documentos a incluir en el análisis, cuya necesidad subyace de una información fiable y considerable para el estudio, puesto que en el análisis de datos cualitativos se busca la emergencia de datos sobre la base de una documentación enriquecedora y con grandes aportes para ser arbitrados en un marco teórico referencial, sucinto de interpretaciones y deducciones de carácter científico (lógico, verificable y sistematizable).

\section{Tercera etapa}

Esclarecer conceptualizaciones operativas para determinar un mismo lenguaje en la creación y alimentación de códigos o atributos de análisis -en notación del software-, como relaciones semánticas derivadas de categorías para el análisis de la teoría a considerar en la investigación. Por ello, este paso es muy importante, pues cada investigador al momento de codificar en las unidades hermenéuticas 
-Espacio o archivo de interpretación en el software- debe tener un concepto claro de ¿Qué se quiere analizar? y ¿Cuál es el origen y definición del código a fundamentar?, sobre todo compartirla con el resto del equipo y poner en práctica dicho lenguaje común en pro de analizar los documentos primarios.

\section{Cuarta etapa}

Luego de codificar y establecer relaciones semánticas, se da paso a fusionar las Unidades Hermenéuticas - unir los archivos interpretativos de cada investigadory así agrupar los datos establecidos y usar un método de comparación constante (Strauss y Corbin, 2002) para realizar la reorganización de las citas, los códigos y memorandos - como elementos que ofrece software para analizar los documentos y el corpus teórico-, a efectos de esclarecer un análisis pertinente para del marco teórico que aborde la problemática del estudio y justifique el problema de investigación.

\section{Resultados}

En esta parte, se pretende ejemplificar y describir el proceso realizado para articular una teoría fundamentada al estudio en cuestión, acoplando los beneficios de articular un proceso informático CAQDAS (Computing Assited Qualitive Data Analysis Software) (Cisneros, 2003), consecuente al desarrollo de un análisis cualitativo, el cual busca reflexionar sobre la importancia de una pertinencia teórico-conceptual subordinando el elemento empírico-tecnológico; no como un fin en los procesos de investigación, sino como una mediación importante para el desarrollo metodológico de los principios epistemológicos inherentes en un marco teórico y referencial a la luz de un objeto de estudio que en este caso; se da en torno a la formación docente en el uso de TIC.

\section{Descripción del proceso cualitativo en el estudio de formación docente en TIC}

Vinculando la etapa uno y dos del método propuesto, el presente escrito se ubica como una investigación de carácter descriptivo. Por ello, su desarrollo se inscribe en un enfoque cualitativo, en tanto los hallazgos como los resultados obtenidos son producto de un ejercicio interpretativo. De igual manera, es necesario asumir que los datos (documentos revisados) corresponden a discursos textuales normalizados con representaciones y visiones susceptibles de ser analizadas, más aún, es labor de los investigadores identificar su sentido e importancia frente a los objetivos de la investigación. Así pues, la investigación en este corte, aboga por estrategias para la clasificación de la información, requiere tanto la evaluación de conceptos definidos previamente, como la identificación de nuevas categorías para la comprensión del fenómeno estudiado sin reduccionismo alguno. La teoría fundamentada se presenta como perspectiva metodológica que orienta la 
clasificación de los documentos, permitiendo ordenar y agrupar la información en unidades analizables.

El uso de la Grounded Theory es útil para esta investigación, por su componente inductivo, cuyo objetivo es construir una tendencia teórica que emerja desde los datos (Glaser y Strauss, 1967; Strauss y Corbin, 2002: 13). Entonces, el estudio gira en torno a la explicación de los diferentes modelos y didácticas concordantes con una formación docente para el uso de las TIC en entornos virtuales de aprendizaje. Entretanto, la emergencia de significados desde los datos hace de esta teoría una metodología adecuada para el conocimiento del fenómeno de formación docente, pues es un objeto que ha sido estudiado desde diferentes perspectivas teóricas (histórica, política, cultural y educativa). Para Strauss y Corbin (2002), la teoría fundamentada puede ser utilizada para un mayor entendimiento de un fenómeno ya estudiado, así poder profundizar en él; como es el caso de la formación como proceso de capacitación y actualización a través de una mediación disciplinar con base en acciones integrales (cognitivas, axiológicas y críticas).

Aunque el propósito de la Teoría fundamentada es la construcción de tendencias teóricas, se busca en este estudio aprovechar las técnicas de codificación y las estrategias de análisis características de esta teoría: la codificación abierta y el método comparativo constante, siendo una alternativa para que los datos puedan ser interpretados con mayor facilidad, por ende, los códigos y su fundamentación -ver apartado metodológico- permitirán entablar significantes; por ejemplo, políticas educativas y modelos pedagógicos asociados e imbricación en la formación docente a nivel de educación superior.

En la codificación abierta se realiza un análisis minucioso, donde el texto se desglosa y se divide en unidades con sentido (Strauss y Corbin, 2002), es decir, unidades significativas para el estudio. Posteriormente, a estas unidades se les asigna un código o concepto (formación docente, herramientas y protocolos en TIC, educación superior, entre otros). Mientras, el método comparativo establece alternantes de datos, en este caso los códigos como unidades de sentido en que se han dividido los textos, identificando similitudes al determinar secuencias en la información, por ejemplo las relaciones entre las características de los docentes que usan los entornos virtuales de aprendizaje como medio de formación y la calidad educativa establecida en este contexto institucional.

Para apoyar los hallazgos en cuanto a datos, se registraron las observaciones, reflexiones e interpretaciones de los codificadores a través de memos o memorandos durante el análisis. Según Strauss y Corbin los memorandos son «el registro que lleva el investigador de los análisis, pensamientos, interpretaciones, preguntas e instrucciones para la recolección adicional de datos» (2002: 21). 


\section{Búsqueda y selección de la información}

La búsqueda de los documentos se realizó a través de bases de datos virtuales especializadas como: Sciencedirect, Scielo, Redalyc, Dialnet, Google académico, entre otras. Además, Se contemplaron 110 documentos de los cuales 40 referían directamente al objeto de estudio: formación docente. En razón a lo anterior, los documentos seleccionados a partir de los criterios de la etapa dos (ver Figura 1), se recopilaron y guardaron como archivos digitales en los formatos .pdf y .rtf, cuyo fin es organizarlos y analizarlos bajo la herramienta o software Atlas.ti.

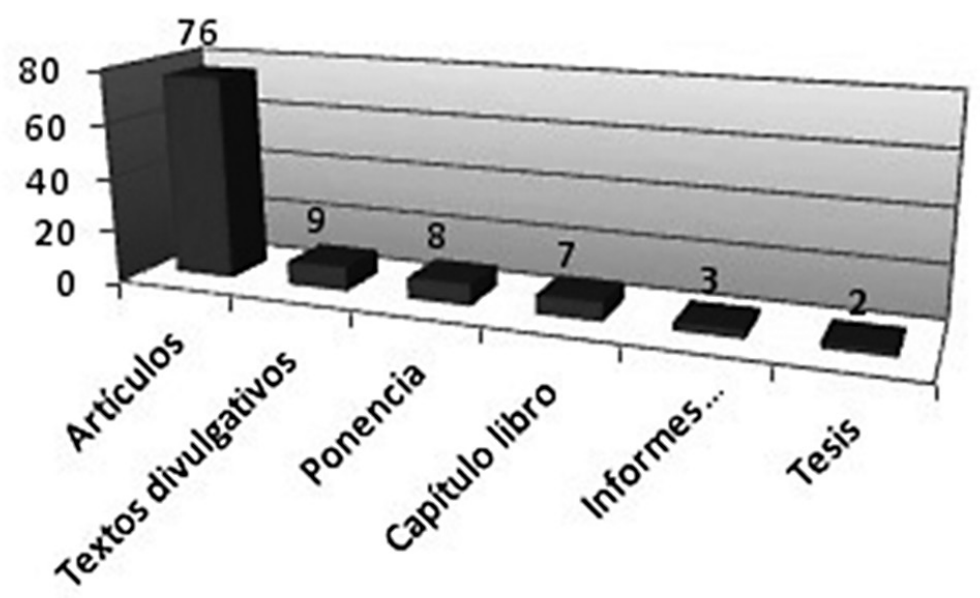

Figura 1. Tipología de documentos primarios utilizados en el estudio.

\section{Proceso de codificación}

De manera previa al proceso de codificación o clasificación de la información, se formularon 6 categorías y 33 subcategorías para que sirvieran como referente en la clasificación de la información. Estas categorías se construyeron desde la discusión y conceptualización de teoría de autoridades académicas en el tema de la formación docente y, producto de investigaciones efectuadas sobre el tema por el grupo de investigadores. Por ende, es muy importante el conocimiento que haya sobre el tema por parte del grupo de investigación. De este modo, en la gráfica se presentan las categorías creadas inicialmente con sus respectivas sub-categorías (ver Figura 2). 


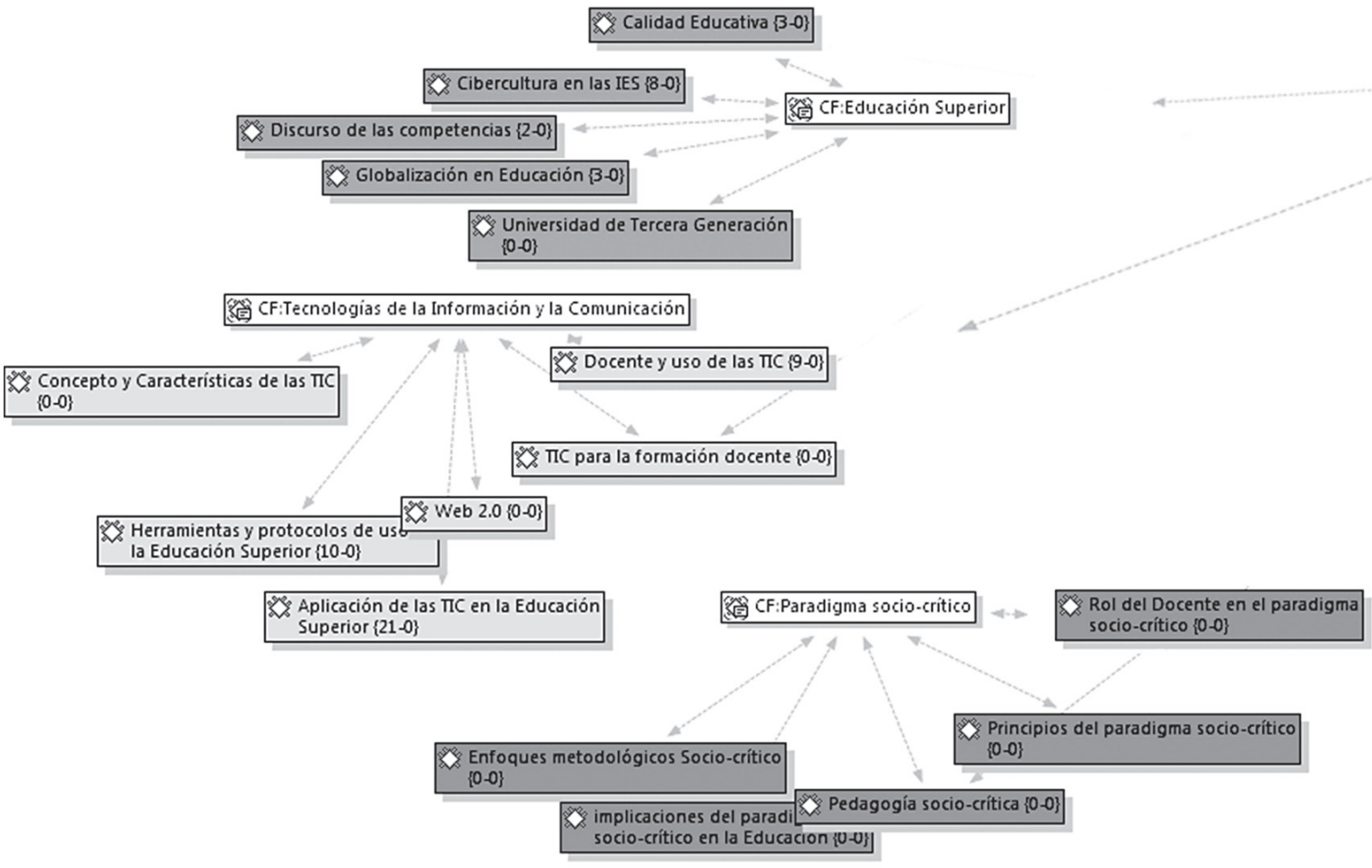

Figura 2. Categoría y sub-categorías del estudio.

También se puede especificar gráficamente la fundamentación de los códigos, a partir de las citas que lo componen (Figura 3), pues, es sumamente relevante determinar cuál código posee mayor citación, porque en una representación e interpretación de los datos, se podría inferir que dicha categoría pudiera conformar el eje de la investigación, estableciendo relaciones de causalidad, asociación, particularidad, entre otras provistas en el software para relacionar diversos códigos en Atlas. ti.

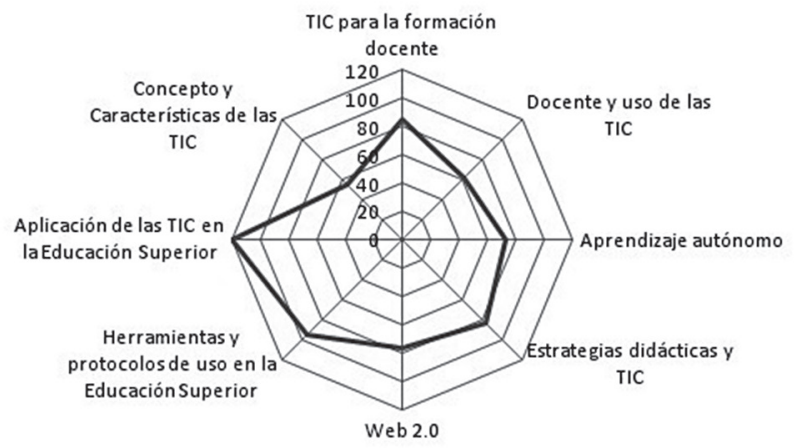

Figura 3. Fundamentación de los códigos respectivos a las principales sub-categorías. 


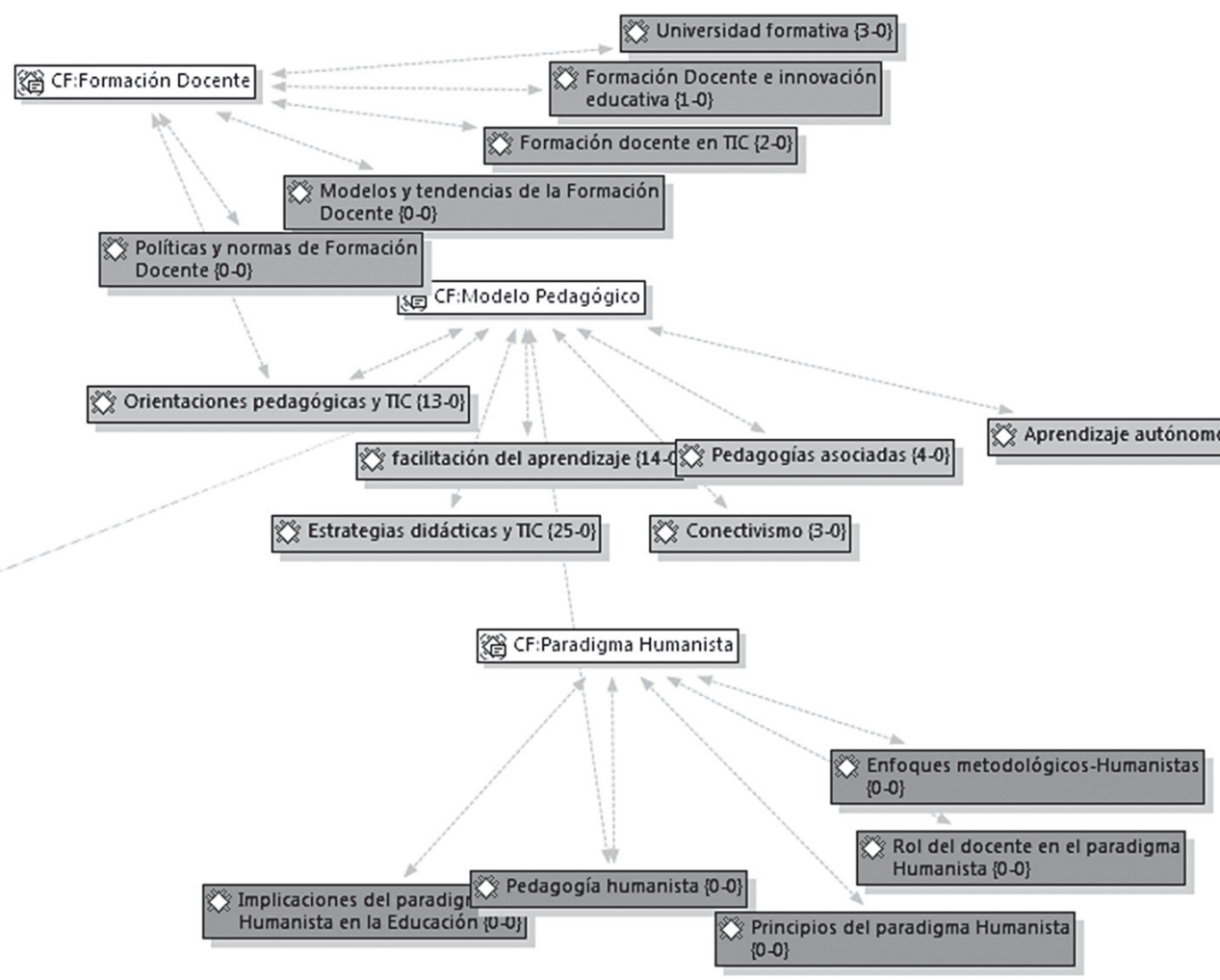

En la primera fase de codificación, se aplicó una clasificación inicial de los datos que permitiera reducir los documentos a unidades analizables, es decir, a fragmentos de texto con significado (citas), cuyo fin es organizar y dotar de sentido las lecturas efectuadas sobre los documentos primarios. Así las cosas, la codificación inicial permitió a los investigadores familiarizarse con el lenguaje, la terminología y la temática de los documentos, lo que posteriormente serviría para evaluar las categorías aplicadas y crear nuevas categorías necesarias para describir con mayor detalle la información.

Como segunda fase en la codificación, se adicionó el método de codificación abierta para construir nuevas categorías que permitieran describir la información. Para éste fin, se realizó la comparación de los códigos y sus respectivas citas, leyendo a profundidad y de ser necesario, ajustando la información en nuevos códigos y jerarquías, permitiendo una mayor comprensión y descripción de las categorías creadas en un principio. Para resumir del proceso hasta ahora mencionado, se muestra el siguiente esquema (Figura 4):

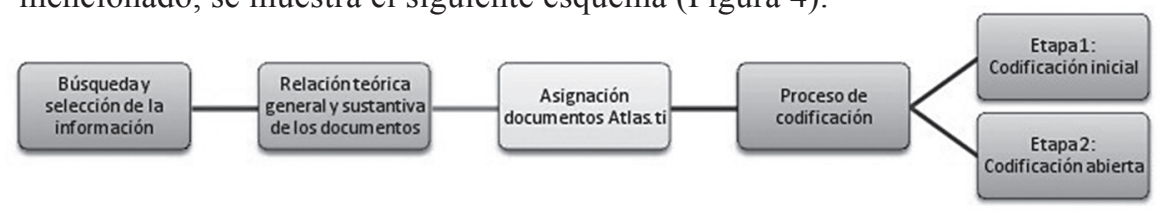

Figura 4. Proceso de análisis y codificación de los documentos primarios. 


\section{Resultados del análisis y clasificación de los datos cualitativos}

La codificación es un proceso muy importante, pues señala la ruta interpretativa para obtener los datos necesarios y así efectuar un análisis riguroso y selectivo sobre el problema objeto de estudio que se está investigando. Ulteriormente, se hace necesario tener representaciones esquemáticas de los datos adquiridos, entonces aparecen los Networks o redes de trabajo (Figura 5), supeditadas a unas áreas de trabajo dispuesta por el software Atlas.ti., para determinar relaciones e interdependencias de citas, códigos, memorandos y muchos otros nodos de análisis cualitativo.

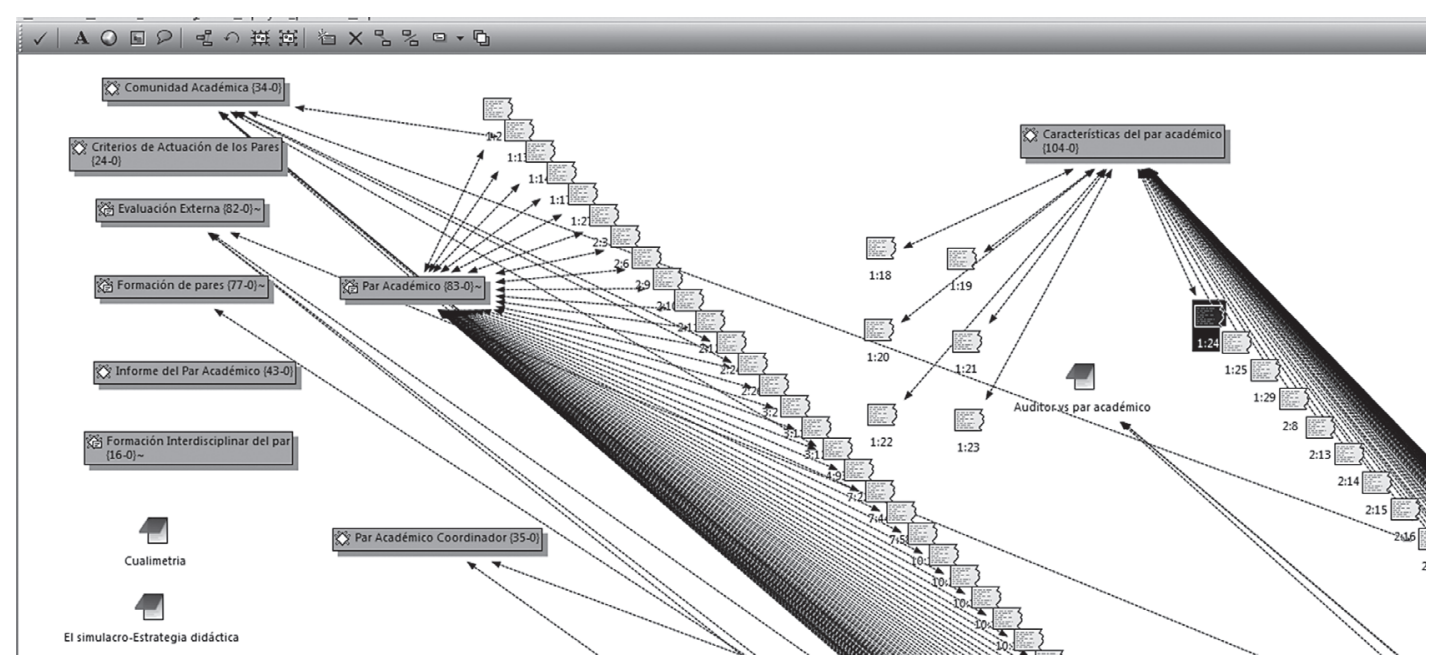

Figura 5. Proceso de análisis y codificación de los documentos primarios. Network y redes semánticas.

En la figura número cinco se muestra cómo se estableció en principio, la relación de los códigos, citas y memorandos de la categoría de formación docente en didáctica basadas en TIC, en la cual, se empieza a establecer posibles conexiones con otros códigos o memorandos y así adquirir información comprensible y con sentido ante el problema que se está indagando. Otra forma de verificar la transversalidad de las codificaciones, es decir, relacionar densidad de códigos y citas, es a través de una tabla de concurrencias (Figura 6), visto como una relación matricial con filtros provistos por Atlas. ti. en aras de visualizar el compartimiento de citas por los códigos. He aquí un ejemplo: 


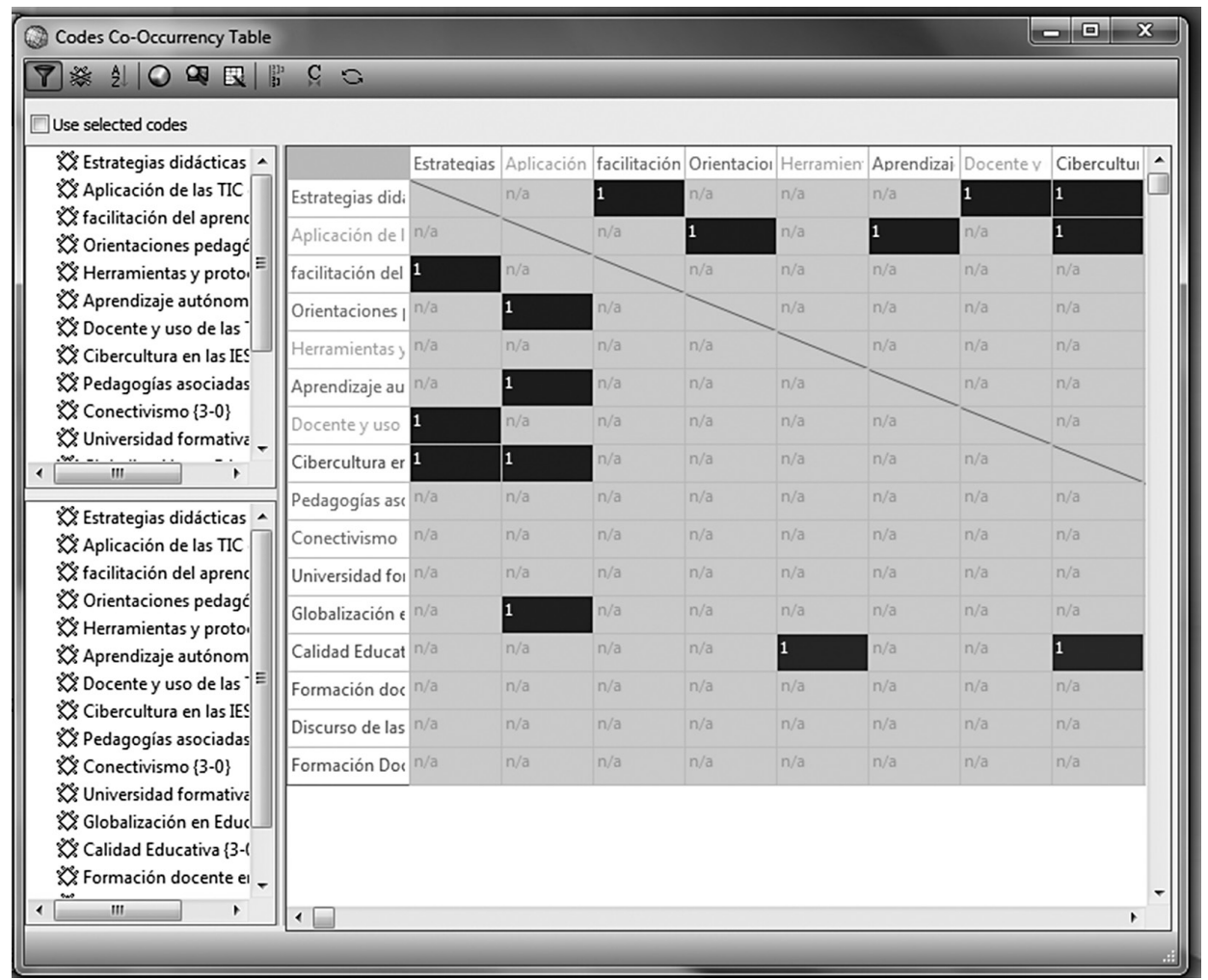

Figura 6. Tabla de concurrencias en Atlas.Ti, versión 6.2.27.

Percibir cuántas citas son compartidas por diversos códigos, ayuda a los investigadores a formular tendencias de reciprocidad, o en otra forma, convalida las relaciones entre categorías y sub-categorías, dando mayor sustento teórico a las relaciones, como también puede romper los preceptos iniciales y llevar por otra ruta al investigador en sus conjeturas al momento de analizar los datos cualitativos. Por último están los sistemas de Output, a manera de mostrar la información clasificada y ordenada con fines de ser mostrada en discusiones grupales o ser comentada a través de mesas de discusión (Figura 7), en tanto muestra la cantidad de citas textualmente con su respectivo autor, en caso de haberse fusionado la Unidades hermenéuticas -ver cuarta etapa del proceso metodológico-, esto contribuye a visualizar lo codificado por cada integrante y hacer comparaciones de fundamentación y densidad entre citas y códigos respectivamente, para llegar a un consenso de elementos conceptuales y desarrollar el marco referencial de la investigación. 


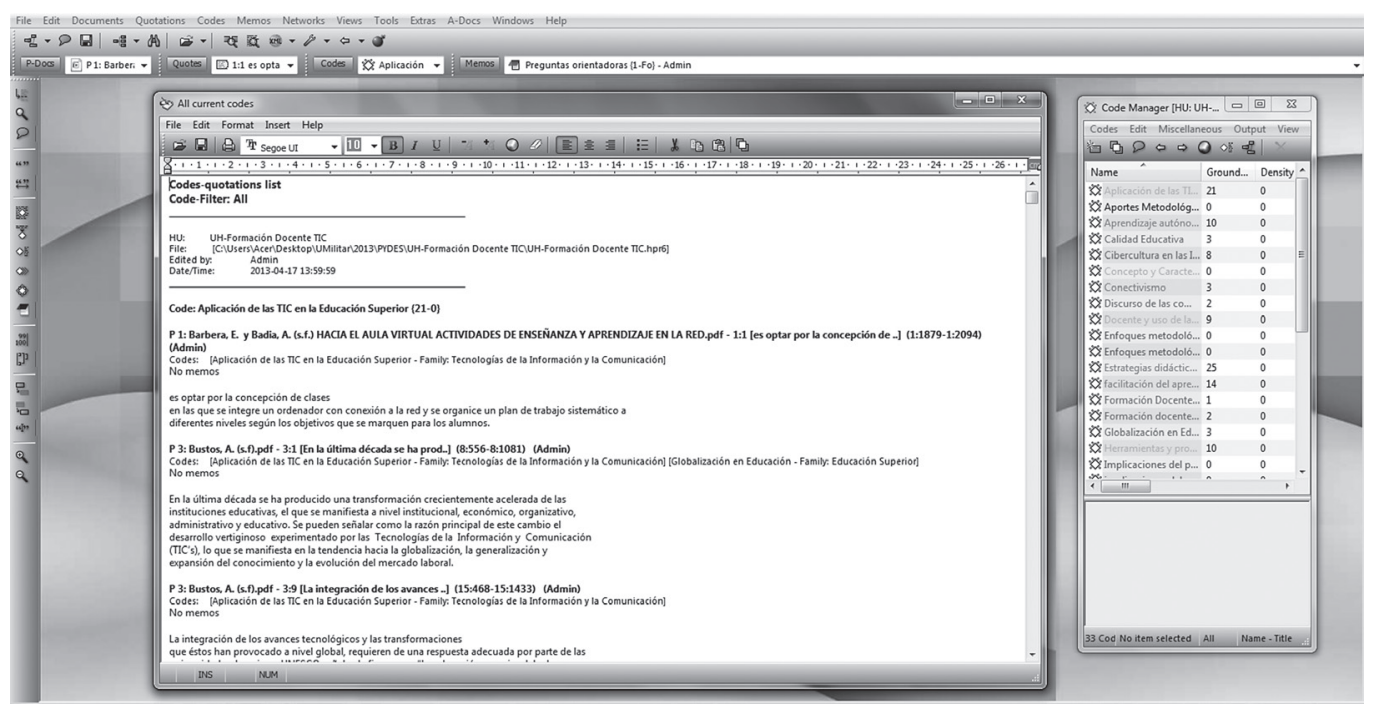

Figura 7. Ejemplo de un Output para las sub-categorías de la investigación.

\section{Conclusiones}

La experiencia de usar el software Atlas.ti. en el marco del proyecto de Formación docente en el uso de las TIC en procesos virtuales de aprendizaje adelantado en la Universidad Militar Nueva Granada, ha permitido reflexionar sobre la importancias de la mediaciones tecnológicas para establecer niveles de análisis, clasificación y depuración de datos de orden cualitativo, aún cuando la investigación o el estudio se muestra desde un enfoque cualitativo. No obstante, es de aclararse que la reflexión gira en torno a desdibujar la idea de que el software es lo imperante, cuando aún más específica es la acción teórica y en el caso de la teoría fundamentada la parte interpretativa, documental y explicativa llevada a cabo por los investigadores para consolidar los datos de forma rigurosa y encontrar significancia en medio de una relación sujeto-objeto y las implicaciones del posicionamiento frente un análisis documental emergente.

La tecnología CAQDAS, entra en vigor siempre y cuando los procesos contemporáneos de investigación a nivel social y más aún en el campo educativo, concedan un análisis riguroso a la construcción de tendencias teóricas en el marco de un posicionamiento epistemológico para investigar el problema abordado, pues con base en la experiencia reflejada en este escrito, se pueden hacer uso del análisis cualitativo mediado por el software, sin recurrir a dicotomías y eclecticismos basados en determinar abstracciones empíricas carentes de sustento teórico, en acopio a los niveles científicos enmarcados en la 
comunidad académica en la cual se inscribe el objeto de estudio. Lo primordial surge cuando estas tecnologías son aplicadas conscientemente a través de la fundamentación teórica como pasa con el software Atlas.ti, articulándose en el estudio por su esencialidad inductiva arraigada en la teoría fundamentada y sus preceptos de comparación constante y codificación abierta, muy importantes para profundizar en aspectos ya trabajados desde otras temporalidades paradigmáticas y perspectivas teóricas.

\section{Referencias bibliográficas}

Cisneros, C. 2003. Análisis cualitativo asistido por computadora. Sociologías, 5 (9): 288313. Disponible en: http://seer.ufrgs.br/sociologias/article/view/5881

Chacón, E. 2004. El uso del ATLAS/TI como herramienta para el análisis de datos cualitativos en investigaciones educativas. Jornadas Universitarias, Competencias socio-profesionales de la titulaciones de Educación. UNED. Disponible en: http://www. uned.es/jutedu/ChaconEdixon-IJUTE-Comunicacion.PDF

Giraldo, M. 2011. Abordaje de la investigación cualitativa a través de la teoría fundamentada en los datos. Actualidad y Nuevas Tendencias. 2 (6): 79-86. Disponible en: http://servicio.bc.uc.edu.ve/ingenieria/revista/Inge-Industrial/VolII-n6/art5.pdf

Glaser, B \& Strauss, A. 1967. The Discovery of Grounded Theory: strategies for qualitative research. New York: Aldine.

Hernández, J, Herrera, L; Martínez, R; Páez, J \& Páez, M. 2011. Generación de teoría. La teoría fundamentada. Universidad del Zulia: Puerto Ordaz. Disponible en: http:// www.eduneg.net/generaciondeteoria/files/INFORME-TEORIA-FUNDAMENTADA.pdf

Murillo, J. s,f. Teoría Fundamentada o Grounded Theory. Madrid: Universidad Autónoma de Madrid.

Sautu, R; Boniolo, P; Dalle, P; \& Elbert, R. 2005. Manual de metodología. Buenos Aires: Colección Campus Virtual

Strauss, A \& Corbin, J. 2002. Bases de la investigación cualitativa. Técnicas y procedimientos para desarrollar la teoría fundamentada. Bogotá: Editorial Universidad de Antioquia.

Valles, M. 2002. Ventajas y desafios del uso de programas informáticos (e.g. ATLAS. ti y MAXqda) en el análisis cualitativo. Una reflexión metodológica desde la grounded theory y el contexto de la investigación social española. Documento de trabajo Serie Sociológica (2001). Fundación de estudios Andaluces.

Valles, M. 2003. Técnicas cualitativas de investigación social, reflexión metodológica y práctica profesional. Madrid: Editorial síntesis. 